\title{
Forecasting Civil Aviation Incident Rate in China Using a Combined Prediction Model
}

\author{
Yixiang $\operatorname{Sun}^{1}$ and Nana Geng $\mathbb{D}^{2}$ \\ ${ }^{1}$ Nanjing University of Aeronautics and Astronautics, Nanjing 211106, China \\ ${ }^{2}$ Nanjing University of Posts and Telecommunications, Nanjing 210023, China \\ Correspondence should be addressed to Nana Geng; shiyu0618@163.com
}

Received 25 February 2021; Revised 20 March 2021; Accepted 1 April 2021; Published 13 April 2021

Academic Editor: di huang

Copyright ( 2021 Yixiang Sun and Nana Geng. This is an open access article distributed under the Creative Commons Attribution License, which permits unrestricted use, distribution, and reproduction in any medium, provided the original work is properly cited.

\begin{abstract}
With the rapid development of air transportation, the complexity, importance, and severity of civil aviation safety have gradually become prominent. It is essential to use various data to analyze and predict the level of aviation safety. This paper used a combined prediction model based on Induced Ordered Weighted Averaging (IOWA) operator to forecast the civil aviation incident rate. We compiled and calculated civil aviation incident data and total flight hours from 2008 to 2019 in China and took the civil aviation incident rate (incident numbers per ten thousand flight hours) as the prediction object. First, this paper used the nonlinear regression model, Grey Verhulst model, and Holt-Winters exponential smoothing model to forecast the civil aviation incident rate individually. Then, it used the smallest sum of squared errors as the principle to use a combined prediction model based on the IOWA operator. It can be seen from the experimental results that the prediction accuracy of the combined model is better than single models. Finally, this paper forecasted the civil aviation incident rate in 2020 and 2021. The results showed that the predicted rates are 0.524 and 0.551 . Most notably the incident rate will increase significantly compared with 2019.
\end{abstract}

\section{Introduction}

Safety is the primary prerequisite for the development of civil aviation. In recent years, the safety level of civil aviation in China has been continuously improved. By the end of September 2020, China civil aviation achieved consecutive safe flight for 86.69 million flight hours and 121 months [1]. The civil aviation accident index in China is better than the world average. However, aviation safety should not be relaxed because accidents in civil aviation will often cause significant losses. The research of aviation accident data and incident data plays a vital role in aviation safety management. Data prediction with scientific algorithms is the key to accident prevention.

Worldwide travel restrictions related to the COVID-19 pandemic caused a sharp drop in the total number of flights. According to reports from Flightradar24, a global flight tracking website, the number of commercial flights in 2020 fell $42 \%$ from the previous year. Nevertheless, crashes of large commercial airliners worldwide caused more deaths than the previous year. According to reports from the Dutch aviation consultancy To70, 299 people died in crashes of civil aviation large commercial airliners in 2020. The number of people who died in crashes of civil aviation large commercial airliners in 2019 is 257 . The aviation operation is full of randomness and uncertainty. So it is essential to strengthen civil aviation data analysis and prediction.

Civil aircraft incident refers to the occurrence of events related to aircraft that do not constitute an accident but may affect safety during the aircraft's operation [2]. The incident rate generally refers to the number of incidents occurring in 10,000 flight hours. So we used the incident rate as the prediction object in this research. More accurate predictions of future civil aviation safety status are conducive to reducing risks and losses before accidents and major accidents occur. Effective prediction can reduce economic losses and has a particular significance for civil aviation safety management development. The civil aviation incident prediction 
methods mainly include single predictions such as regression prediction, time series prediction, and Grey prediction and combination predictions of Grey Markov prediction and intelligent algorithm models such as SVM and BP. In actual prediction, different methods can be used to predict the same problem. Improving the forecasting accuracy of incident rate has an important practical significance for aviation practitioners to judge aviation safety trends and formulate correct safety strategies.

This paper used a combined prediction model for forecasting civil aviation incident rates in the next two years based on IOWA operator. First, based on the incident rate data from 2008 to 2019, we used the nonlinear regression model, Grey Verhulst model, and Holt-Winters exponential smoothing model to forecast the aviation accident rate individually. Then, we established a combined prediction model based on the IOWA operator with the smallest sum of squared errors as the principle. Finally, we forecasted the incident rate of civil aviation in China from 2020 to 2021.

\section{Literature Review}

At present, scholars' research on civil aviation safety data analysis mainly focuses on the following aspects:

(1) The monitoring or recognition of a certain kind of event, risk analysis, or related model research. For example, Korsun and Poplavskii [3] estimated the measurement error of the aircraft angle of attack using satellite navigation data. Huang et al. [4] proposed a feature extraction method for distinguishing abnormal flight events. Sun and Han [5] proposed a method for analyzing Quick Access Recorder (QAR) overrun events and flight record data using a different test, which can solve the massive QAR Information with guiding significance extracted from flight data. Qi et al. [6] studied the high-risk areas that may trigger the QAR overrun event using the QAR record data and the golden section method. Sun and Meng [7] used the cluster analysis method to study aviation flight incidents' time distribution law and obtained a short period of high aviation incidents. Liu and He [8] studied the flight safety risk index based on the frequency and intensity of flight parameter overrun. Geng [9] used QAR data to analyze pilots' long-term performance and carried out quantitative evaluation methods for their technical flight level. Wang et al. [10] used flight QAR data to study heavy landing accidents and established a quantitative risk assessment model for heavy landing using a statistical modeling method. Jian et al. [11] presented a risk assessment method for civil aviation flight safety based on QAR overrun events.

(2) Use relevant data for fault diagnosis and trend prediction. Some researchers have used a variety of forecasting models to forecast passenger traffic $[12,13]$. Shan [14] used the Bayesian network to predict civil aviation unsafe events and made noise diagnosis and high-risk identification for unsafe events. Dai [15] analyzed the management mode and relevant civil aviation flight quality monitoring measures and analyzed the civil aviation flight quality monitoring and early warning management. Wang et al. [16] used the Auto Regressive Moving Average (ARMA) model to predict tail collision events in domestic civil aviation transportation. Sun et al. [17] proposed a spatiotemporal analysis method of flight quality monitoring based on temporal Geographic Information System (GIS). Du [18] used exponential function to perform regression analysis on civil aviation accident rate data from 1993 to 2007 and conducted a correlation test. Liu et al. [19] used correlation analysis to choose five positively related influencing factors. They adopted air transport incidents' historical data and their influencing factors to establish air transportation incidents' cluster prediction model. Wang et al. [20] proposed a nonlinear regression prediction model of civil aviation incidents based on taking off and landing sorties. Bin et al. [21] proposed a combined prediction model based on GM (1.1) and Markov model to forecast the civil aviation incidents based on the incident data and operational aircraft data from 2003 to 2012. Wang and $\mathrm{Li}$ [22] analyzed civil aviation incidents' characteristics and proposed a series of grey neural network combined prediction models. Xiong et al. [23] used the Long Short-Term Memory (LSTM) neural network model to train and predict the bird strike incidents data. Chen et al. [24] proposed a combined prediction model based on Autoregressive Integrated Moving Average (ARIMA) and Back Propagation Neural Networks (BPNN) to forecast civil aviation incidents in other months. Liang and Li [25] used the combined model of ARIMA, Least Squares Support Vector Machines (LS-SVM), and BPNN to forecast and analyze the airline company's monthly flight incidents rate. Valdés et al. [26] developed five Bayesian models of increasing difficulty to predict and anticipate incidents. Subramanian and Rao [27] used LSTM networks to forecast incident data derived from the National Aeronautics and Space Administration's Aviation Safety Reporting System incident database. The forecast generated helps identify factors that contribute significantly to the trends seen in multiple categories of incidents and also provides insight into which categories of incidents are more (or less) likely to occur in the forecast period.

It can be seen from the literature review that econometric models have been widely used in civil aviation event prediction, such as the ARIMA model, exponential smoothing model, and regression model. The Grey prediction model has good applicability in the prediction of a civil aviation incident. Most of them focus is on optimizing GM $(1,1)$ model in many aspects and applying it to predicting the civil aviation incident. As intelligent algorithms 
can solve traditional prediction models' limitations, more and more intelligent algorithm technologies have been applied to predicting civil aviation incidents, including BP algorithm, RBF algorithm, genetic algorithm, etc. In large data samples, intelligent algorithms have more advantages, and in the case of small data samples, traditional models such as the Grey model have good accuracy. In general, the combination prediction models have attracted more and more attention because of their better prediction accuracy.

\section{Combined Prediction Model of Civil Aviation Incident Rate Based on IOWA Operator}

The civil aviation industry's development is full of uncertainties and challenges, so predicting with a single model may not achieve good results. It is possible to consider combining multiple types of prediction methods appropriately to comprehensively utilize multiple models' information to improve the prediction's reliability and accuracy. It is the principle of the combined prediction model. The IOWA operator can give weight according to the prediction accuracy of each prediction model at each time and establish a combined prediction model with the sum of square error as the objective function [28-30]. IOWA operator theory is introduced as follows [31].

3.1. Ordered Weighted Averaging (OWA) Model. Assume $f_{\text {owa }}: R^{m} \longrightarrow R$ is the function with $m$ variables; thus,

$$
f_{\text {owa }}\left(a_{1}, a_{2}, \ldots, a_{m}\right)=\sum_{i=1}^{m} w_{i} b_{i}
$$

$W=\left(w_{1}, w_{2}, w_{3}, \ldots, w_{m}\right)^{T}$ is the weight vector related to the function $f_{\text {owa }}$, where

$$
\sum_{i=1}^{m} w_{i}=1, \quad w_{i} \geq 0, i=1,2, \ldots, m
$$

Resort the sequence $a_{1}, a_{2}, \ldots, a_{m}$ from the largest to the smallest and get a new sequence; $w_{i}$ is related to the order of $a_{1}, a_{2}, \ldots, a_{m}$.
3.2. IOWA Operator. Assume there are $m$ two-dimensional arrays, $\left\langle u_{1}, a_{1}\right\rangle,\left\langle u_{2}, a_{2}\right\rangle, \ldots,\left\langle u_{m}, a_{m}\right\rangle, \quad$ and $f_{\text {iowa }}: R^{m} \longrightarrow R$ is the function with $m$ variables; thus,

$$
f_{\text {iowa }}\left(\left\langle u_{1}, a_{1}\right\rangle,\left\langle u_{2}, a_{2}\right\rangle, \cdots,\left\langle u_{m}, a_{m}\right\rangle\right)=\sum_{i=1}^{m} w_{i} a_{u-\operatorname{index}(i)} .
$$

Here, $u_{1}, u_{2}, u_{3}, \ldots, u_{m}$ are the induced values and $u-$ index $(i)$ is the subscript of the $i-$ th number in $a_{1}, a_{2}, \ldots, a_{m}$ arranged in descending order. $w_{i}$ is not related to the size and position of $a_{1}, a_{2}, \ldots, a_{m}$, but it is related to the location of the induced value.

3.3. Combined Prediction Model Building Process. If $m$ kinds of single prediction method are used to predict the observation value of an indicator sequence $\left\{y_{t}, t=1,2, \ldots\right\}$, then the prediction accuracy is

$$
u_{i t}=\left\{\begin{array}{l}
1-\left|\left(x_{t}-x_{i t}\right) / x_{t}\right|, \quad\left|\left(x_{t}-x_{i t}\right) / x_{t}\right|<1 \\
0, \quad\left|\left(x_{t}-x_{i t}\right) / x_{t}\right| \geq 1 .
\end{array}\right.
$$

$i=1,2, \ldots, m$ means the number of prediction method, $t=$ $1,2, \ldots, N$ means time, $u_{i t}$ means the prediction accuracy, and $u_{i t} \in[0,1], x_{t}$ is the actual value and $x_{i t}$ is the prediction value. Assume $u_{i t}$ is the induced value of $x_{i t}$, and the prediction accuracy and the prediction value form a two-dimensional array $\left[u_{1 t}, x_{1 t}\right],\left[u_{2 t}, x_{2 t}\right],\left[u_{3 t}, x_{3 t}\right], \ldots,\left[u_{i t}, x_{i t}\right]$.

Arrange $u_{1 t}, u_{2 t}, u_{3 t}, \ldots, u_{m t}$ from the largest to the smallest, and mark the $i$-th prediction accuracy as $u$-index (it); thus, the combined prediction value is

$$
f\left[\left(u_{1 t}, x_{1 t}\right),\left(u_{2 t}, x_{2 t}\right), \ldots,\left(u_{m t}, x_{m t}\right)\right]=\sum_{i=1}^{m} w_{i} x_{u-\operatorname{index}(i t)} .
$$

Assume $e_{u \text {-index }(i t)}=x_{t}-x_{u \text {-index }(i t)}$. If the experimental standard is specified as the smallest sum of squared errors, the model can be rewritten as

$$
\begin{aligned}
& \min S(L)=\sum_{i=1}^{m} \sum_{j=1}^{m} w_{i} w_{j}\left(\sum_{t=1}^{N} e_{u-\operatorname{index}(i t)} e_{u-\operatorname{index}(j t)}\right), \\
& \text { s.t. }\left\{\sum_{i=1}^{m} w_{i}=1, w_{i} \geq 0, \quad i=1,2, \ldots, m .\right.
\end{aligned}
$$

3.4. Evaluation of Prediction Model. Prediction accuracies are usually compared using the following four indicators:

Mean square error (MSE):

$$
\mathrm{MSE}=\frac{1}{n} \sum_{t=1}^{n}\left(\widehat{x}_{t}-x_{t}\right)^{2}, \quad t=1,2, \ldots, n .
$$

Root mean square error (RMSE):

$$
\text { RMSE }=\sqrt{\frac{1}{n} \sum_{t=1}^{n}\left(\widehat{x}_{t}-x_{t}\right)^{2}, \quad t=1,2, \ldots, n .}
$$

Mean absolute error (MAE):

$$
\mathrm{MAE}=\frac{1}{n} \sum_{t=1}^{n}\left|\widehat{x}_{t}-x_{t}\right|, \quad t=1,2, \ldots, n .
$$


Mean absolute percentage error (MAPE):

$$
\text { MAPE }=\frac{1}{n} \sum_{t=1}^{n}\left|\frac{\widehat{x}_{t}-x_{t}}{x_{t}}\right|, \quad t=1,2, \ldots, n .
$$

In the above four evaluation index expressions, $x_{t}$ is the actual value at time $t, \widehat{x}_{t}$ is the predicted value of the model at time $t$, and $n$ is the number of forecast periods.

\section{Forecasting Civil Aviation Incident Rate in China}

4.1. Date Source. According to relevant data $[32,33]$, the necessary data of civil aviation incidents and civil aviation flight hours in China from 2008 to 2019 are selected.

It can be seen from Table 1 that the civil aviation incident rate showed a clear increasing trend before 2018, from 0.303 in 2008 to 0.554 in 2017, and the rate decreased from 2018. It shows that civil aviation development direction in China is shifting from rapid expansion to high-quality development. Civil aviation safety management, especially the analysis and prediction of accidents and incidents, is necessary for such a critical period.

4.2. Nonlinear Regression Model Prediction. According to relevant data $[32,33]$, the necessary data of civil aviation incidents in China, civil aviation flight hours, and civil aviation incidents per 10,000-hour rates from 2008 to 2019 are selected. A nonlinear regression model is a commonly used prediction method. Due to the randomness of civil aviation events, the number and frequency data are relatively discrete. The reasonable degree of linear regression is insufficient, but the reasonable degree of nonlinear regression models such as exponential function, power function, and polynomial is good. We use power function regression, exponential function regression, logarithmic function regression, polynomial regression, and other nonlinear regressions to establish the prediction models. Since the error is the smallest and the fitting degree is the highest, the power function regression is selected to establish the power function regression prediction model:

$$
y=2.8393 \times 10^{-287} \times x^{86.6185846} .
$$

According to the above formula, the prediction result and error and accuracy are calculated, shown in Table 2. Comparison of power function regression forecast data and actual data is shown in Figure 1.

4.3. Grey Verhulst Model Prediction. The Grey Verhulst model combines the specific advantages of the grey prediction theory and the Verhulst model. Based on the Verhulst model's whitening equation, the difference equation's parameters and the differential equation have an excellent agreement through a series of mathematical derivation. Simultaneously, it applies the sample with insufficient information and little data and has good prediction feasibility.
TABle 1: Civil aviation incident rate in China from 2008 to 2019.

\begin{tabular}{cccc}
\hline Year & Incident rate & Year & Incident rate \\
\hline 2008 & 0.303 & 2014 & 0.424 \\
2009 & 0.324 & 2015 & 0.463 \\
2010 & 0.384 & 2016 & 0.547 \\
2011 & 0.363 & 2017 & 0.554 \\
2012 & 0.477 & 2018 & 0.492 \\
2013 & 0.437 & 2019 & 0.463 \\
\hline
\end{tabular}

TABle 2: The prediction and fitting data of the power function regression model.

\begin{tabular}{lcccc}
\hline Years & Actual data & Forecast data & Error & Accuracy \\
\hline 2008 & 0.303 & 0.342 & -0.039 & $87.13 \%$ \\
2009 & 0.324 & 0.357 & -0.033 & $89.81 \%$ \\
2010 & 0.384 & 0.373 & 0.011 & $97.14 \%$ \\
2011 & 0.411 & 0.389 & 0.022 & $94.65 \%$ \\
2012 & 0.477 & 0.406 & 0.071 & $85.12 \%$ \\
2013 & 0.437 & 0.424 & 0.013 & $97.03 \%$ \\
2014 & 0.424 & 0.443 & -0.019 & $95.52 \%$ \\
2015 & 0.463 & 0.462 & 0.001 & $99.78 \%$ \\
2016 & 0.547 & 0.483 & 0.064 & $88.30 \%$ \\
2017 & 0.554 & 0.504 & 0.05 & $90.97 \%$ \\
2018 & 0.492 & 0.526 & -0.034 & $93.09 \%$ \\
2019 & 0.463 & 0.549 & -0.086 & $81.43 \%$ \\
\hline
\end{tabular}

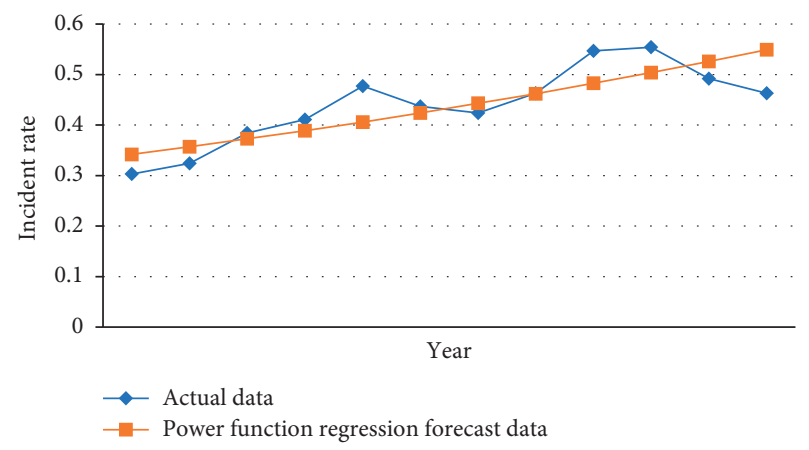

Figure 1: Comparison of power function regression forecast data and actual data.

Establish the nonnegative original sequence:

$$
X^{(0)}=\left(x^{(0)}(1), x^{(0)}(2), \ldots, x^{(0)}(n)\right),
$$

and one-time accumulation sequence is

$$
X^{(1)}=\left(x^{(1)}(1), x^{(1)}(2), \ldots, x^{(1)}(n)\right),
$$

where

$$
X^{(1)}(k)=\sum_{i=1}^{k} X^{(1)}(k), \quad k=1,2, \ldots, n,
$$

and background value sequence is

$$
Z^{(1)}=\left(z^{(1)}(2), z^{(1)}(3), \ldots, z^{(1)}(n)\right)
$$

where 


$$
z^{(1)}(k)=\frac{1}{2}\left(x^{(1)}(k-1)+x^{(1)}(k)\right), \quad k=2,3, \ldots, n .
$$

The basic expression form of the Grey Verhulst model is

$$
X^{(0)}(k)+a z^{(1)}(k)=b\left(z^{(1)}(k)\right)^{2} .
$$

The parameter estimation vector of the model is $\widehat{v}=(a, b)^{T}$. Thus, the least squares estimation of $X^{(0)}(k)+$ $a z^{(1)}(k)=b\left(z^{(1)}(k)\right)^{2}$ is $\widehat{v}=(a, b)^{T}=\left(B^{T} B\right)^{-1} B^{T} Y$, where

$$
B=\left[\begin{array}{c}
-z^{(0)}(2)\left(z^{(0)}(2)\right)^{2} \\
-z^{(0)}(3)\left(z^{(0)}(3)\right)^{2} \\
\vdots \\
-z^{(0)}(n)\left(z^{(0)}(n)\right)^{2}
\end{array}\right], Y=\left[\begin{array}{c}
x^{(0)}(2) \\
x^{(0)}(3) \\
\vdots \\
x^{(0)}(n)
\end{array}\right] .
$$

The whitening differential equation of the Grey Verhulst model is

$$
\frac{\mathrm{d} x^{(1)}}{\mathrm{d} t}+a x^{(1)}=b\left(x^{(1)}\right)^{2} .
$$

The time response function can be obtained as

$$
x^{(1)}(t+1)=\frac{a x^{(1)}(0)}{b x^{(1)}(0)+\left(a-b x^{(1)}(0) e^{a t}\right)} .
$$

Discretize the albino differential equation and take the initial value condition: $\hat{x}^{(1)}(1)=x^{(1)}(1)$.

The time response sequence of the Grey Verhulst model can be obtained as

$$
X^{(1)}(k+1)=\frac{a x^{(1)}(1)}{b x^{(1)}(1)+\left(a-b x^{(1)}(1)\right) e^{a k}}, \quad k=1,2, \ldots, n .
$$

Finally, the predicted and fitted values of the Grey Verhulst model can be obtained by reduction of formula.

The parameters $a=-0.286$ and $b=-0556$ can be obtained through calculation.

The time response sequence of the Grey Verhulst model is

$$
\widehat{x}^{(1)}(k+1)=\frac{-0.087}{-0.168+-0.117 e^{-0.286 k}}, \quad k=1,2, \ldots, n .
$$

The prediction and fitting data of the Grey Verhulst model are shown in Table 3 and Figure 2.

4.4. Exponential Smoothing Model Prediction. The exponential smoothing model is an essential time series analysis method, which is also called the exponential weighted average method. The exponential smoothing model has been widely used in practice because of its characteristics, such as simple operation, convenient calculation, and excellent prediction performance.
TABLE 3: The prediction and fitting data of the Grey Verhulst model.

\begin{tabular}{lcccc}
\hline Years & Actual data & Forecast data & Error & Accuracy \\
\hline 2008 & 0.303 & 0.303 & 0 & $100 \%$ \\
2009 & 0.324 & 0.337 & -0.013 & $96.14 \%$ \\
2010 & 0.384 & 0.369 & -0.015 & $96.09 \%$ \\
2011 & 0.411 & 0.397 & 0.014 & $96.59 \%$ \\
2012 & 0.477 & 0.421 & 0.056 & $88.26 \%$ \\
2013 & 0.437 & 0.441 & 0.004 & $99.09 \%$ \\
2014 & 0.424 & 0.457 & -0.033 & $92.78 \%$ \\
2015 & 0.463 & 0.47 & -0.007 & $98.51 \%$ \\
2016 & 0.547 & 0.48 & 0.067 & $87.75 \%$ \\
2017 & 0.554 & 0.488 & 0.066 & $88.09 \%$ \\
2018 & 0.492 & 0.494 & -0.002 & $99.60 \%$ \\
2019 & 0.463 & 0.499 & -0.036 & $92.79 \%$ \\
\hline
\end{tabular}

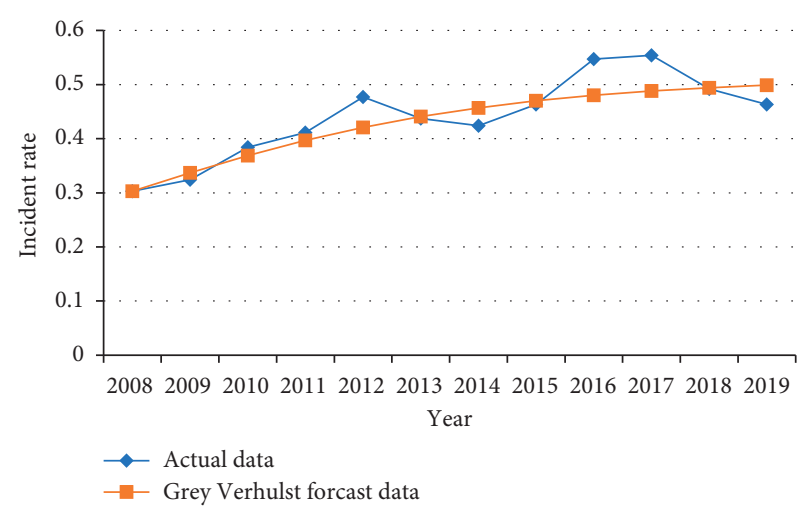

Figure 2: Comparison of Grey Verhulst model forecast data and actual data.

Holt-Winters Model was first proposed by Winters in 1960, and later, through the continuous improvement of Cipra, Romera, Hyndman, etc., the existing model formula was gradually formed. Basically, the Holt-Winters model is a cubic exponential smoothing approach, which is one of the standard models for time series analysis.

In the process of modeling the Holt-Winters model, the sample sequence is first decomposed into three parts: linear trend, trend increment, and seasonal change, and then the three components are estimated separately using the exponential smoothing method, and finally the extrapolation is established separately modeling and obtaining the extrapolated predicted value of the sample sequence.

The Holt-Winters nonseasonal model is suitable for the trend prediction problem without the influence of seasonal changes. The mathematical expression of the model is as follows:

$$
\left\{\begin{array}{l}
S_{t}=\alpha X_{t}+(1-\alpha)\left(S_{t-1}-b_{t-1}\right), \\
b_{t}=\gamma\left(S_{t}-S_{t-1}\right)+(1-\gamma) b_{t-1}, \\
F_{t+m}=S_{t}+m b_{t} .
\end{array}\right.
$$

The Holt-Winters additive model is suitable for time series forecasting problems with seasonal additive changes. The mathematical expression of the model is as follows: 


$$
\left\{\begin{array}{l}
S_{t}=\alpha\left(X_{t}-I_{t-L}\right)+(1-\alpha)\left(S_{t-1}-b_{t-1}\right) \\
I_{t}=\beta\left(X_{t}-S_{t}\right)+(1-\beta) I_{t-L} \\
b_{t}=\gamma\left(S_{t}-S_{t-1}\right)+(1-\gamma) b_{t-1} \\
F_{t+m}=S_{t}+m b_{t}+I_{t-L+m} .
\end{array}\right.
$$

The Holt-Winters multiplication model is suitable for time series forecasting problems with multiplicative seasonal changes. The mathematical expression of the model is as follows:

$$
\left\{\begin{array}{l}
S_{t}=\alpha\left(\frac{X_{t}}{I_{t-L}}\right)+(1-\alpha)\left(S_{t-1}-b_{t-1}\right), \\
I_{t}=\beta\left(\frac{X_{t}}{S_{t}}\right)+(1-\beta) I_{t-L}, \\
b_{t}=\gamma\left(S_{t}-S_{t-1}\right)+(1-\gamma) b_{t-1}, \\
F_{t+m}=\left(S_{t}+m b_{t}\right) I_{t-L+m} .
\end{array}\right.
$$

In the formula, $X_{t}$ is the actual value of the civil aviation incident rate; $F_{t+m}$ is the predicted value of the civil aviation incident rate for period $m ; F_{t+m}$ is the length of the season; $\alpha$, $\beta, \gamma$ are smoothing parameters, and the value range is a closed interval of $0 \sim 1 ; b_{t}$ is trend component, and the initial value is $0 ; S_{t}$ is stable component at time $t$, and the initial value is $S_{t} \sum_{t=1}^{L}\left(X_{t} / L\right)$; $I_{t}$ is the seasonal component at time $t$, and the initial value of $I_{t}$ in the additive model is $X_{t}-S_{t}$. The initial value of $I_{t}$ in the additive model is $X_{t} / S_{t}$.

In this paper, EViews software is used to establish a HoltWinters Exponential Smoothing Model. The initial smoothing value is the default value of the system. Alpha, Beta, and Gamma values are automatically selected by EViews software to minimize the sum of squares between the actual value of the sequence and the sequence's smoothing value. For the Holt-Winters model, its parameter estimation is shown in Table 4.

At this point, the smoothing parameter $\alpha$ is $0.36000, \beta$ is $1.0000, \gamma$ is 0.0000 , and the error sum of squares is 0.004171 .

The prediction and fitting data of the Holt-Winters model are shown in Table 5 and Figure 3.

4.5. Combination Prediction Model. Establish a two-dimensional array:

$$
\left[a_{1 t}, x_{1 t}\right],\left[a_{2 t}, x_{2 t}\right],\left[a_{3 t}, x_{3 t}\right], \ldots,\left[a_{m t}, x_{m t}\right] .
$$

Calculate the IOWA combination forecast value as follows:

$$
\begin{aligned}
& f_{L}\left(\left[a_{11}, x_{11}\right],\left[a_{21}, x_{21}\right],\left[a_{31}, x_{31}\right]\right)=0.342 L_{1}+0.303 L_{2}+0.320 L_{3} \\
& f_{L}\left(\left[a_{12}, x_{12}\right],\left[a_{22}, x_{22}\right],\left[a_{32}, x_{32}\right]\right)=0.357 L_{1}+0.337 L_{2}+0.316 L_{3}
\end{aligned}
$$

Then use LINGO software to get the weight of each individual forecast:

$$
\begin{aligned}
& w 1=0.9999998, \\
& w 2=1.42 \mathrm{E}-08, \\
& w 3=7.35 \mathrm{E}-09 .
\end{aligned}
$$

Constructing the combination prediction model with this weight, the calculated results are shown in Table 6 and Figure 4 . The average prediction accuracy of the combined prediction model is $96.92 \%$.

4.6. Prediction Model Evaluation. As shown from the result figures of the prediction model, both the Grey Verhulst model and the power function model are monotone curves, and their fitting curves are relatively stable. However, neither of these two models can reflect the fluctuation of data. So, when the incident rate fluctuates significantly in the years of 2012 and 2016, the error is relatively large. The exponential smoothing model curve can reflect the fluctuation of the actual curve. The incident rate is highly dependent on the recent data and has obvious trend change, but there is always a certain distance between the actual value and the predicted value. Combined prediction weights and averages the predicted values of each individual forecast at each time point, and the result is highly correlated with the prediction accuracy at each time point. It is a dynamic combination of three single forecast models. Combination forecasting is more coincident with the factual data.

To compare and analyze the prediction effects of different models, we use the four indicators of MSE, RMSE, $M A E$, and MAPE to evaluate the models. Calculate the four evaluation indicators of the three single prediction models, and compare them with the combination prediction model. The comparison results are shown in Table 7.

It can be seen from the four error evaluation indexes that the error evaluation of the combined forecasting model is less than that of the single forecasting method. Because the combined prediction effectively integrates the sample information and selects the advantages of a single prediction, the fitting accuracy is also higher than that of the general combined prediction model. Due to the randomness of events, certain important factors may be ignored when using a particular forecasting method. Careful consideration of several forecasting methods and operators' use as a combined forecasting model will improve the forecasting accuracy.

4.7. Forecast the Incident Rate. Use the combination prediction model to predict the civil aviation incident rate of the 
TABle 4: The prediction and fitting data of the Grey Verhulst model.

\begin{tabular}{lc}
\hline Alpha & 0.3600 \\
\hline Beta & 1.0000 \\
\hline Gamma & 0.0000 \\
\hline Sun of squared residuals & 0.004171 \\
\hline Root mean squared error & 0.018643 \\
\hline
\end{tabular}

TABle 5: The prediction and fitting data of the Holt-Winters model.

\begin{tabular}{lcccc}
\hline Years & Actual data & Forecast data & Error & Accuracy \\
\hline 2008 & 0.303 & 0.320 & -0.017 & $94.39 \%$ \\
2009 & 0.324 & 0.316 & 0.008 & $97.53 \%$ \\
2010 & 0.384 & 0.363 & 0.021 & $94.53 \%$ \\
2011 & 0.411 & 0.424 & -0.013 & $96.84 \%$ \\
2012 & 0.477 & 0.458 & 0.019 & $96.02 \%$ \\
2013 & 0.437 & 0.427 & 0.010 & $97.71 \%$ \\
2014 & 0.424 & 0.444 & -0.020 & $95.28 \%$ \\
2015 & 0.463 & 0.496 & -0.033 & $92.87 \%$ \\
2016 & 0.547 & 0.496 & 0.051 & $90.68 \%$ \\
2017 & 0.554 & 0.531 & 0.023 & $95.85 \%$ \\
2018 & 0.492 & 0.499 & -0.007 & $98.58 \%$ \\
2019 & 0.463 & 0.486 & -0.023 & $95.03 \%$ \\
\hline
\end{tabular}

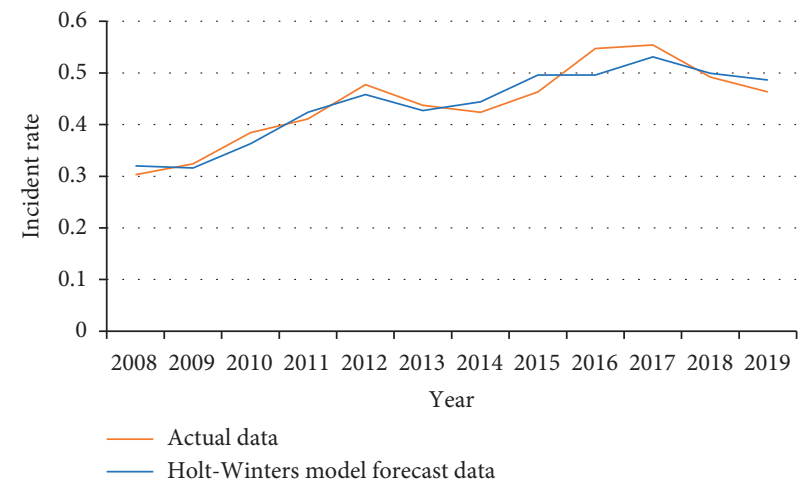

Figure 3: Comparison of Holt-Winters model forecast data and actual data.

TABLE 6: The prediction and fitting data of the combined prediction model.

\begin{tabular}{lccc}
\hline Years & Actual data & Forecast data & Accuracy \\
\hline 2008 & 0.303 & 0.303 & $100.00 \%$ \\
2009 & 0.324 & 0.316 & $97.53 \%$ \\
2010 & 0.384 & 0.373 & $97.14 \%$ \\
2011 & 0.411 & 0.424 & $96.84 \%$ \\
2012 & 0.477 & 0.458 & $96.02 \%$ \\
2013 & 0.437 & 0.441 & $99.08 \%$ \\
2014 & 0.424 & 0.443 & $95.52 \%$ \\
2015 & 0.463 & 0.462 & $99.78 \%$ \\
2016 & 0.547 & 0.496 & $90.68 \%$ \\
2017 & 0.554 & 0.531 & $95.85 \%$ \\
2018 & 0.492 & 0.494 & $99.59 \%$ \\
2019 & 0.463 & 0.486 & $95.03 \%$ \\
\hline
\end{tabular}

year 2020 and 2021. Significantly, the combined prediction model based on IOWA Operator takes the arithmetic mean of all the weights as the weight of the forecast moments when

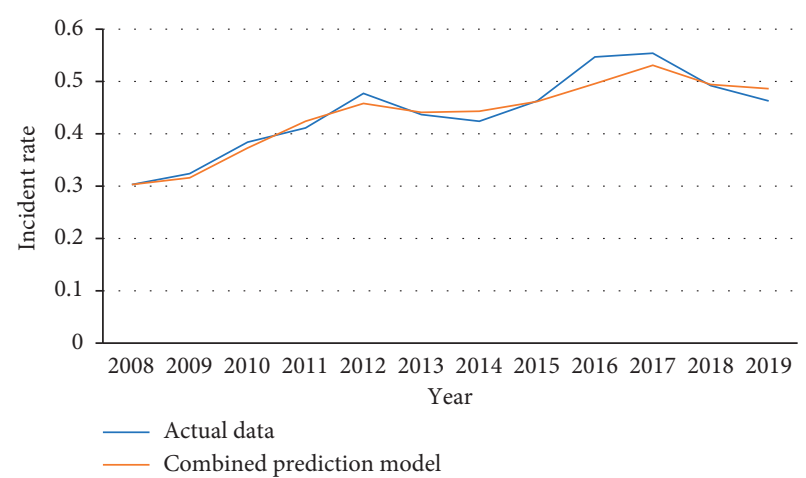

FIgURE 4: Comparison of combined prediction model forecast data and actual data.

TABLe 7: Comparison of combination prediction model and single prediction models.

\begin{tabular}{lcccc}
\hline Model & MSE & RMSE & MAE & MAPE \\
\hline $\begin{array}{l}\text { Power function } \\
\text { regression }\end{array}$ & 0.001995 & 0.044661 & 0.036917 & 0.083368 \\
Grey Verhulst & 0.001252 & 0.035384 & 0.026083 & 0.054683 \\
Holt-Winters & 0.000552 & 0.023495 & 0.020417 & 0.045578 \\
Combined prediction & 0.000396 & 0.019908 & 0.014500 & 0.030785 \\
\hline
\end{tabular}

TABle 8: Civil aviation incident rates of years 2020 and 2021.

\begin{tabular}{lll}
\hline Year & 2020 & 2021 \\
\hline Combined prediction model & 0.524 & 0.551 \\
\hline
\end{tabular}

determining the future prediction weight coefficient. When determining each forecast model's weight during the forecast period, the simple average method is used to determine the three single forecast models' weights in the forecast period. After calculation, during the forecast period, the weights of the Power Function Regression model, Grey Verhulst model, and the Holt-Winters model are $w_{1}=0.1816, w_{2}=0.1819, w_{3}=0.6365$. The predicted value of the civil aviation incident rate in the next two years by the combined forecasting model based on the IOWA operator is calculated. The prediction results are shown in Table 8.

According to the combined model's prediction results, civil aviation incident rates in 2020 and 2021 will be 0.524 and 0.551 , showing a rising trend. This prediction result is contrary to the civil aviation incident rate in China which has declined for two consecutive years from 2018 to 2019. Simultaneously, considered with the aviation industry's current situation under the influence of the COVID-19, aviation safety needs more concerns.

\section{Conclusions}

Based on the theoretical research on civil aviation incident rate forecasting, this paper used a combined prediction model based on IOWA operator to overcome the shortcomings of the single prediction model and applies it to the 2020-2021 civil aviation incident rate prediction. Using the incident rate instead of the number of incidents to predict 
civil aviation's safety level can weaken incidents' randomness and have higher stability. It can reflect the safety level of civil aviation more accurately.

Regarding the reasons for the possible increase in incident rate, we hold that, on the one hand, the aviation industry is currently in a difficult period, and aviation safety management may be lax. On the other hand, due to the COVID-19 epidemic, many uncertain factors have been caused, which further leads to safety issues. COVID-19 has brought considerable changes to the aviation industry and brought new safety risks. The total number of flights will continue to be affected by worldwide travel restrictions. It has a significant impact on the flight crew and other aviation industry-related employees. Many employees were suspended from work. Aviation safety issues under the influence of the COVID-19 pandemic deserve more attention and further discussion. For example, after the aviation industry's recovery, flight attendants' "skill decline" may become a critical problem.

The aviation industry must take preventive measures to control new safety risks. We all hope to sweep away the haze of the past, and reducing the rate of civil aviation incidents becomes one of the goals. To effectively maintain aviation safety management, we should increase the investigation and management of potential safety hazards and strengthen the control of critical risks. In addition to strengthening flight attendants' skill training, it is equally essential for countries to resolve international conflicts and reduce potential risks peacefully.

\section{Data Availability}

All data generated or analyzed during this study are included within this article.

\section{Conflicts of Interest}

The authors declare no conflicts of interest.

\section{Acknowledgments}

This work was supported by the Nanjing University of Posts and Telecommunications research start-up fund (NY219168), the Nature Foundation Incubation Fund of Nanjing University of Posts and Telecommunications (NY220214), the Project of Philosophy and Social Science Research in Colleges and Universities in Jiangsu Province (TJZ220011), and the Jiangsu Innovation Program for Graduate Education (KYLX15_0312).

\section{References}

[1] Information Office of the State Council, China's Civil Aviation Development has made Six Achievements During the 13th Five Year Plan, Information Office of the State Council, Beijing, China, 2020, http://n.eastday.com/ pnews/1603361709021070,2020-10-22/2020-12-10.

[2] MH/T (2001-2018), “Civil aircraft incident sign,” 2018.

[3] O. N. Korsun and B. K. Poplavskii, "Estimation of systematic errors of onboard measurement of angle of attack and sliding angle based on integration of data of satellite navigation system and identification of wind velocity," Journal of Computer and Systems Sciences International, vol. 50, no. 1, pp. 130-143, 2011.

[4] Y. Huang, J. Wang, and B. Gu, "Feature extraction method of QAR data based on ball vector machine," Informatization Research, vol. 23, pp. 107-108, 2010.

[5] R. Sun and W. Han, "Parameter characteristics analysis of flight overrun events based on difference test," China Science and Technology of Work Safety, vol. 10, pp. 10-11, 2011.

[6] L. Qi, X. Shao, and H. Chi, "Flight operation risk diagnosis method for QAR overrun event," Journal of Beijing University of Aeronautics and Astronautics, vol. 36, pp. 17-18, 2011.

[7] R. Sun and L. Meng, "Study on time distribution of aviation flight incidents," Traffic Information and Safety, vol. 2, no. 31, pp. 83-87, 2013.

[8] X. Liu and Y. He, "Flight safety quantitative evaluation model fram-fd based on FDR," Journal of University of Electronic Science and Technology of China. 2006, vol. 35, no. 1, 2013.

[9] S. Geng, "Research on pilot flight technology evaluation method based on QAR data," Tianjin: Civil Aviation University of China, vol. 08, pp. 14-17, 2007.

[10] L. Wang, R. Sun, and C. Wu, "Heavy landing risk quantitative evaluation model based on flight QAR data," Chinese Journal of Safety Sciences, vol. 24, no. 2, pp. 88-92, 2014.

[11] W. Jian, Z. Xia, and J. Wang, "Civil aviation flight safety risk assessment method based on QAR overrun event," Science and Technology Guide, vol. 37, no. 11, pp. 101-108, 2019.

[12] J. Zhou, X. Kuang, and Z. Chen, "A demand forecasting model for air passenger traffic in China: based on the stochastic frontier analysis model and model averaging," Systems Engineering-Theory \&, vol. 40, no. 11, pp. 117-127, 2020.

[13] J. Biay, A. Rurak, and W. K. J. Tomasze, "Statistical analysis of the volume of passenger air traffic in Europe in years 2004-2015," Transportation Research Procedia, vol. 35, pp. 72-79, 2018.

[14] J. Shan, Prediction Reasoning and Risk Identification of Civil Aviation Unsafe Event Data, Beijing Jiaotong University, Beijing, China, 2016.

[15] L. Dai, "Analysis of civil aviation flight quality monitoring and early warning management," Dual Use Technologies and Products, vol. 14, 2017.

[16] L. Wang, X. Yang, and S. Gao, "Prediction of civil aviation tail scraping events based on time series model," Safety and Environmental Engineering, vol. 27, no. 2, pp. 216-220, 2020.

[17] H. Sun, Y. Liu, and Y. Chen, "Spatial temporal analysis of QAR big data based on Temporal GIS," Computer Engineering and Design, vol. 39, no. 3, pp. 694-700, 2018.

[18] Y. Du, "Regression analysis of the 10,000-hour rate of civil aviation incidents," Journal of Civil Aviation Flight University of China, vol. 1, pp. 42-44, 2010.

[19] L. Liu, Y. Sun, and H. Zheng, "Prediction model of the air transport incidents of civil aviation based on set pair analysis," Safety and Environmental Engineering, vol. 20, no. 5, pp. 154-158, 2013.

[20] Y. Wang, J. Zhu, and N. Ga, "Nonlinear regression prediction of civil aviation incidents based on taking off and landing sorties," Journal of Wuhan University of Technology (Information and Management Engineering), vol. 38, no. 6, pp. 672-677, 2016.

[21] W. Bin, "Realization of GMPC model in incident prediction," Science and Technology in Western China, vol. 13, no. 4, pp. 17-30, 2014.

[22] Y. Wang and H. Li, "Research on prediction model of civil aviation accident symptomatic based on grey neural 
network," China Safety Science Journal, vol. 22, no. 3, pp. 10-15, 2012.

[23] M. Xiong, H. Wang, and Y. Xu, "General aviation safety research based on prediction of bird strike symptom," Systems Engineering and Electronics, vol. 42, pp. 2033-2040, 2020.

[24] F. Chen, D. Zhang, and W. Wei, "Integrated forecasting model for the civil aviation incidents rate based on the ARIMA and BP neural network," Journal of Safety and Environment, vol. 6, pp. 2040-2047, 2019.

[25] W. Liang and X. Li, "Pre-diction of air traffic accidents based on ARIMA, LS-SVM and BP neural network models," Safety and Environ-Mental Engineering, vol. 25, no. 1, pp. 130-136, 2018.

[26] R. M. A. Valdés, V. F. G. Comendador, and L. P. Sanz, "Prediction of aircraft safety incidents using Bayesian inference and hierarchical structures - ScienceDirect," Safety Science, vol. 104, pp. 216-230, 2018.

[27] S. V Subramanian and A. H Rao, "Deep-learning based time series forecasting of go-around incidents in the national airspace system," in Proceedings of the 2018 AIAA Modelling and Simulation Technologies Conference, Grapevine, TX, USA, January 2018.

[28] Q. Chen and H. Chen, "Optimal combination forecasting model based on two kinds of criteria of Iowa operator and its application," Mathematical Statistics and Management, vol. 5, pp. 91-97, 2013.

[29] H. Zhang, Q. Chen, and C. Xiong, "Optimal grey combination model based on Iowa operator and its application," Statistics and Decision, vol. 32, no. 174, pp. 75-79, 2017.

[30] D. Huang, X. Chen, Z. Liu, C. Lyu, S. Wang, and X. Chen, "A static bike repositioning model in a hub-and-spoke network framework," Transportation Research Part E: Logistics and Transportation Review, vol. 141, Article ID 102031, 2020.

[31] H. Chen and C. Liu, "Combination forecasting method based on Iowa operator," Forecasting, vol. 22, no. 6, pp. 61-65, 2003.

[32] Civil Aviation Administration of China, China Air Transport Development Report, Civil Aviation Administration of China, Beijing, China, 2009.

[33] Civil Aviation Administration of China, Civil Aviation Industry Development Statistics Bulletin, Civil Aviation Administration of China, Beijing, China, 2019. 